Published in: Tissue engineering using ceramics and polymers,

Woodhead Publishing Ltd (31 Oct 2007), by A.R. Boccaccini (Author), J. Gough (Author)

ISBN 1845691768

\title{
Carrier systems and biosensors for biomedical applications
}

Frank Davis and Séamus P. J. Higson ${ }^{\dagger}$

Cranfield Health, Cranfield University, Silsoe, MK45 4DT, UK.

${ }^{\dagger}$ Corresponding author email, s.p.j.higson@cranfield.ac.uk.

\section{Introduction}

This chapter addresses both carrier systems and biosensors which are often applied directly to tissues, either as skin patches, implanted or ingested by a variety of routes. It follows that there is a common theme between these applications and many of those discussed elsewhere within this book. Any device, scaffold or implant within the body must usually display extreme biocompatibility if it is not to cause harm to the patient. The techniques of tailoring surfaces to ensure no adverse reactions are a common theme running throughout this work on tissue engineering..

The first section of this chapter will describe the use of carrier systems in biomedical applications. Initially a discussion is provided as to why carrier systems are required and this is followed by descriptions of different classes of material of natural and synthetic origin. This first section will continue with a description of how nanotechnology is becoming utilised in carrier systems and will close with a discussion of current and future applications of these materials. 
The second section of this chapter will be devoted to biosensors, beginning with a history and descriptions of basic sensor formats. Glucose biosensors will be described in more detail due to their dominance of the biosensor market. The construction of first, second and third generation biosensors and their principle of operation will be described. This will be followed by a description of the work progressing to the latest devlopments for implantable glucose sensors and their potential for continuous glucose monitoring. The chapter will close with a description of future needs including warfarin monitoring and implantable organs.

\section{Carrier systems}

\subsection{Introduction}

In many instances a simple one-off administration of a drug will suffice, e.g. taking an analgesic in response to a headache. However this simple approach is often insufficient since often the condition may require continuous medication over a period of time, with many conditions requiring long term drug therapy. Often a drug may have an optimum concentration within the body; too low and no benefit is derived, too high and unwanted side effects or toxicity can occur, endangering the health of the patient. However when a drug is ingested or injected, the level within the patient's blood tends to rise up towards a plateau and then after some time falls again to zero unless more drug is given. In many instances a more efficient application method would be one that gives a stable level of treatment. Other problems can occur with drug stability, for example for a drug to be taken orally it must not be degraded by digestive processes. At the time of writing approximately $15 \%$ of the current world pharmaceutical market consists of products which utilise a carrier system and the US 
market for drug delivery systems is expected to reach $\$ 82$ billion by 2007 (Roco and Bainbridge 2002).

To minimise drug degradation and optimise drug delivery, a wide variety of drug carrier and delivery systems are under investigation and several reviews have been published on this subject (Langer 1995, 1998, Kaparissides et al 2006).

A number of methods can be utilised to apply various carrier systems:

Ingestion - the drug can be incorporated into a carrier system so that the composite material can be swallowed. This method is the most popular due to its simplicity and convenience. The carrier system must be capable of protecting the drug against the highly acidic medium of the stomach and/or enzymatic degradation throughout the digestive system. Sometimes the digestive process itself can be utilised to cause release of the drug. There are, however, a number of problems with this technique since it can lead to irritation of the bowel and also in many cases the drug must still penetrate the stomach/intestine wall.

Inhalation - the drug can be dispersed within an aerosol, usually via a nebuliser and in this way can be directly inhaled. Especially suitable for treating respiratory diseases, this technique is widely used for the treatment of asthma. However delivery via this method can still be adversely affected by the barrier between air and blood within the lung. 
Transdermal - the drug is incorporated within a patch, similar to the nicotine patches used to relieve "cravings" of people attempting to stop smoking. This method avoids the problems of degradation of drugs by digestive process and can be used to provide local delivery e.g. to a wound or skin conditions. Patches often need to be applied only once every several days and the method is non-invasive and painless. It also has the advantage that unlike oral and inhalation routes, patches can be easily and safely applied to unconscious patients. The skin barrier does lead to slow penetration rates and therefore in many instances only relatively low dosage levels can be attained. Other problems may include a lack of dosage flexability.

Injection - this technique has the disadvantage of being invasive and can be painful. Drug carrier systems can be utilised within this method, for example to prevent degradation of the drug within the bloodstream. As an alternative, a drug/carrier composite can be surgically implanted close to an affected site.

\subsection{Classes of materials}

\subsubsection{Hydrophilic polymers}

The pharmaceutical industry has shown great interest in the development of controlled release systems based on hydrophilic polymers. Hydrogels represent a common class of polymers used for drug delivery. In essence a hydrogel is a material based on polymers such as poly(vinyl alcohol) or poly(acrylic acid) which would normally be soluble in water. However either during or after the polymer synthesis, a degree of crosslinking converts the linear polymer chains into a polymer network. This process renders the polymers insoluble, however the high presence of hydrophilic groups within the network gives the structure a high affinity for water. 
Although the network does not dissolve, it is capable of adsorbing water with consequent swelling of the polymer matrix. The nature of the polymer and degree of crosslinking affects the swelling behaviour. A network with few crosslinks and a large number of hydrophilic groups will adsorb large amounts of water with a high degree of swelling. Less hydrophilic monomers, incorporation of hydrophobic co-monomers or a high degree of crosslinking all act to reduce water adsorption, usually leading to a firmer, more rigid firmer gel. Due to the fact that they include a high water content, hydrogels often show high degrees of biocompatibility. The polymer network itself can be either bio-inert or be a biodegradable polymer network. Natural polymers can also be used, with for example, hydrogels based on chitosan, alginase or collagen having been utilised. The application of a wide variety of hydrophilic biodegradable polymers to delivery of proteins has been extensively reviewed (Gomboltz et al 1995). Typical polymers include hydrogels based on poly(vinyl alcohol), poly(vinylpyrrolidinone) or cellulose and other natural polymers such as alginase or collagen. Frequently the drugs, especially if they are biologically derived (such as proteins) can be quite unstable. Besides enabling the controlled release of the active material, these hydrogels often act as a stabilizing media for these unstable agents. For example a drug could be incorporated into a hydrogel either as it is synthesized or post-synthesis. This can then be applied to the patient via any of the techniques described above. Once in vivo, the drug is released by a number of means. These might include simple diffusion or alternatively the polymer may be eroded or dissolved, for example, by digestion. In an ideal situation this process will occur at a constant rate leading to continual release of controlled amounts of the drug. If the polymer is utilised as a transdermal patch or is surgically implanted close to an affected site, the drug can be delivered where it is most needed. 
A similar method utilises polymers which are actually degraded rather than just swollen. Examples include polymers which are based on poly(lactide) or poly(glycolide), (Figure 1), and which are slowly hydrolysed in vivo to release an active agent, e.g. leuprolide acetate (Ogawa et al 1988).

A polymer must address several criteria before it is suitable for use as a carrier system. In the case of polymers that are effective in vivo, they must be both biocompatible and must biodegrade within a reasonable period of time. Irrespective of the manner of application, the polymer itself and any degradation products must be non-toxic and must create neither any allergic or inflammatory response. The method of release is often dependant on the nature of the drug itself; low molecular weight drugs are capable of diffusing out of the polymeric matrix and if water soluble, will be rapidly released. However larger molecules such as proteins will not diffuse as readily and often remain within the hydrogel matrix until the polymer itself is either degraded or enzymatic digestion releases them. Release rates depend on several factors including the quantity/dosing of drugs within the composite, the rate of degradation of the polymer, the water content, (if it is a hydrogel type material), and the presence and degree of crosslinking.

A property that makes hydrogels exceedingly suitable for drug encapsulation is that many of the materials used for these systems can be synthesised so as to be responsive to their environment. A change of $\mathrm{pH}$ for example, can lead to protonation or deprotonation of active groups within the polymer. This can change its affinity for water, giving us a material whose swelling is $\mathrm{pH}$ responsive, thereby affecting many 
of their other physical properties such as permeability. This gives us the opportunity to design drug release agents which are selectively triggered by certain conditions - e.g. their responsiveness to $\mathrm{pH}$ means they can be designed to release active agents within a selected part of the digestive tract. Similar smart materials can be designed which respond to changes in physiological conditions and therefore only release the drug at times when it is needed. Hydrogels can be designed which respond to other stimuli as well as $\mathrm{pH}$, such as ionic strength, temperature and electric field. They can even be designed to respond to the presence or absence of specific analytes.

As mentioned earlier, polymer-based delivery systems allow application of active agents in many ways including ingestion, transdermal patches, suppositories, ocular and subcutaneous methods. A few examples are given here: Nitroglycerin is often used in the treatment of angina, however volatilization of the active component can lead to loss of tablet activity (Markovich et al 1997). This problem can be mitigated by the use of a acrylic-based hydrogel as a host for the nitroglycerin and incorporation of this composite in the construction of a transdermal patch.

There has been wide research into utilising these materials in the treatment of cancer for, for example the delivery of ara-C for leukaemia. This drug gives rise to a number of side effects but these are mitigated when a constant infusion of the drug is introduced subcutaneously. As an alternative approach, crosslinked polyhydroxyethyl acrylate can be utilised as a host for this material. Discs of this composite display a steady controllable release of the active material (Teijon et al 1997). A similar composite based upon polycaprolactone/polyethylene glycol has been used as a 
matrix for the anticonvulsant drug clonazepam. Stable constant release properties were displayed for over 45 days (Cho et al 1999).

Similar polymers are suitable for localised delivery of pharmaceuticals. In the treatment of brain cancer, one approach that has been successfully used is surgery to remove as much of the tumour as possible, followed by placing in the surgical site, small wafers based on polyanhydrides. These contain the anti-cancer drug carmustine which is slowly released over a one month period to kill any remaining tumour cells (Brem et al 1995). Alternatively thermosensitive hydrogels based on block copolymers of poly(ethylene oxide) and poly(lactide) have been made which are liquid at $45^{\circ} \mathrm{C}$ and can be injected directly to the required site. Upon cooling to body temperature, the gel immediately sets, trapping any pharmaceutical compounds in the solution, so allowing them to be released slowly (Jeong et al 1997).

The materials mentioned so far are usually based on simple polymeric systems, however, more complex reactive systems can be designed. As mentioned earlier, hydrogels can be synthesised which respond to environmental conditions. An example of this is a "smart" porous membrane made from a polymethacrylic acid/polyethylene glycol copolymer. This is used as a host for insulin but also contains encapsulated glucose oxidase. Glucose oxidase specifically catalyses the oxidation of glucose to gluconic acid, which causes a subsequent $\mathrm{pH}$ drop. This leads to shrinkage of the membrane and the controlled release of insulin in response to hyperglycaemia (Gander et al 2001). Apart from drug delivery, recent work has focussed on the use of hydrophilic polymers in gene therapy and has shown that encapsulation of the DNA 
inside a hydrophilic polymer can increase the transfection efficiency (Ottenbrite 1999).

\subsection{Natural polymers}

Besides synthetic polymers, natural polymers have also been widely studied as drug carrier agents, especially as for many of them their biocompatibility and non-toxicity are well known since they form major components of our diets. Proteins are of great interest due to their wide variety of structures and their easy availability from sources such as, for example, egg-white, soybean and whey (Chen et al 2006). Hydrogels can be easily generated from a wide variety of proteins and possess the desirable qualities of their synthetic analogues. Usually proteins can be gelated by methods such as heating, which unfolds the polypeptide chains within the protein structure. These then tend to aggregate forming a three dimensional structure crosslinked by hydrogen bonding and/or hydrophobic effects (Clark et al 2001). Other methods for inducing gelation include crosslinking with calcium ions (Maltais et al 2005). A wide variety of hydrogel morphologies and microstructures can be generated by variation of the preparation technique (Chen et al 2006). Applications of these gels include controlled release of tocopherol (Chen et al 2006) from an lactoglobulin based emulsion under simulated gastric conditions. Other examples include the incorporation of drug compounds within albumin (Sokolowski and Roger 1984, Tomlinson and Burgen 1985) or corn protein (Liu et al 2005) and a recent review has been published on this topic (Chen et al 2006). 


\subsection{Micelles, vesicles and liposomes}

Micelles are formed by a wide range of amphiphilic surfactant molecules in aqueous solution. A typical amphiphile contains a long alkyl chain and a polar headgroup. In solution the interactions between the alkyl chains and water are extremely unfavourable and this drives aggregation of the alkyl chains together to minimise this interaction. A structure then forms as shown schematically in Figure 2, where a spherical aggregate spontaneously assembles, with the polar headgroups on the outside of the sphere and a hydrophobic interior, stabilised by Van der Waals interactions between the chains.

What makes micelles so useful as carrier agents is that hydrophobic species can be incorporated within the core of the micelle. This enables the transport of hydrophobic active agents at concentrations much higher than would be possible for a simple aqueous solution of these compounds. The hydrophobic environment of the core moreover protects the guest against hydrolysis and enzymatic degradation.

As an alternative to the classical long chain surfactants shown in Figure 2, amphiphilic block copolymers are also very adept at forming micellar structures. For example a block copolymer containing a hydrophobic block such as polystyrene and a hydrophilic block such as polyethylene oxide, can dissolve in water to give a micellar structure with a polystyrene core surrounded by a polyethylene oxide corona, with the whole structure being typically $5-50 \mathrm{~nm}$ in diameter. Block copolymers re especially suitable for this purpose since they are available in a wide variety of molecular weights. Since a wide variety of monomers and the composition ratios can easily be selected, this allows fine control of the size, composition and morphology of the 
micelles. Incorporation of many functional groups is easily attained; for example crosslinking groups can be utilised to stabilise the micelle structure and the surface properties, selectivity and biocompatibility of the micelles can all be tailored.

In a similar manner, a variety of compounds can be utilised to form vesicles. These are somewhat more complex structures than micelles (figure 2) in which a spherical membrane is formed containing an aqueous core. Liposomes are types of vesicles which are widely used within drug transport and consist of spherical phospholipid bilayers with an aqueous core and are the most commonly used of these carrier systems (Langer 1998, Kaparissides et al 2006). They are capable of transporting either hydrophilic agents within the core - or hydrophobic agents which are incorporated into the membrane. The membrane itself can be tailored to prevent access of enzymes to the core, thereby preventing enzymatic degradation, but do however allow the diffusion out of the active agent. For example channel type proteins may be incorporated within the membrane and retain their activity, thereby allowing transport of ions or other small solutes such as drugs through the membrane.

The use of liposomes can lead to a large increase in drug carrying capacity compared to the use of polymeric systems, however disadvantages can be encountered such as shorter shelf-life and rapid destruction of the vesicle within the body. The biocompatabilty of these systems however can be improved by modifying their surface properties, such as by the attachment of polyethylene glycol units (Park et al 1997, Lasic et al 1999, Moghimi et al 2003). Also, antibodies can be attached to the surfaces of these systems, allowing them to specifically target sites that require treatment. Tumours, for example can be targeted by substituting liposomes with 
antibodies against the HER2 proto-oncogene, found in breast and other cancers (Kirpotin et al 1997, Park et al 1998). Similarly amylopectin can be attached to the surface of liposomes to enable targeting of lung tissue (Poanini et al 1997).

\subsection{Nanotechnology}

In recent times, nanotechnology has become an intensely studied field of research. The application of nanosized materials is expected to make significant advances in biomedical applications such as drug delivery and gene therapy (Sahoo and Labhasetwar 2003, Moghimi et al 2001, 2003).

Earlier in this chapter the use of biodegradable polymers as drug carriers was discussed. Nanoparticles of these types of materials with sizes in the range 10-1000 $\mathrm{nm}$ can be synthesised and incorporate drug molecules by way of entrapment or binding. Similarly, nanocapsules can be synthesised in which a polymer membrane forms a vesicle-like structure with the drug molecules confined within a central cavity. Because of their small size, these systems are capable of passing through small capillaries and be taken up by cells, allowing efficient drug accumulation at a target size (Desai et al 1997). In these materials size and surface properties determine their distribution in the body (Sahoo and Labhasetwar 2003). Localised application of these nanoparticles can be achieved by their tendency to accumulate in tumours due to enhanced permeation effects; this has been extensively reviewed elsewhere (Maeda 2001). Alternatively the nanoparticles can be delivered locally to the size of interest such as within a specific artery after balloon angioplasty (Guzman et al 1996) to deliver long term release (14 days) of dexamethasone. 
Dendrimers are branched polymers grown from a central core with very precisely controlled degrees of polymerisation, Figure 3 shows a schematic of a fourth generation dendrimer. This makes their size, composition and molecular controllable to an exact degree and is an alternative form of polymer nanoparticle to those made by classical emulsion type polymerisations. There has been great interest in utilising dendrimers as drug carrier agents because not only can the bulk of the dendrimer be synthesised exactly but a wide variety of surface groups can be utilised, enabling further surface functionalisation. Dendrimers for example can be made with hydrophilic surfaces and hydrophobic interiors or vice versa. Recent reviews on this subject (Svenson and Tomalia 2005, Gupte et al 2006) extensively detail many of the most pertinent advances in this field so only a few highlights will be given here.

Dendrimers have been utilized to carry a variety of small molecule pharmaceuticals. Commercial PAMAM dendrimers have been used to encapsulate the anticancer drug cisplatin, giving conjugates that exhibited slower release, higher accumulation in solid tumours, and lower toxicity compared to the free drug (Malik et al 1999). PAMAM dendrimers which had been functionalised with poly(ethylene glycol) chains had their encapsulation behaviour for the anticancer drugs adriamycin and methotrexate studied. Up to 6.5 adriamycin molecules or 26 methotrexate molecules per dendrimer could be incorporated for one of the materials studied. The drug release from this dendrimer was slow at low ionic strength but fast in isotonic solution (Kojima et al 2000). Similar dendrimers encapsulated the anticancer drug 5-fluorouracil, showing reasonable drug loading, and reduced release rate and haemolytic toxicity (Bhadra et al 2003). Up to 78 molecules of the anti-inflammatory drug ibuprofen molecules were complexed by PAMAM dendrimers through electrostatic interactions between the 
dendrimer amines and the carboxyl group of the drug. The drug was successfully transported into lung epithelial carcinoma cells by the dendrimers (Kohle et al 2003). Other recent studies have also indicated that low generation PAMAM dendrimers cross cell membranes (El-Sayed et al 2003).

Ceramic nanoparticles have also been studied due to their inherent advantages of ease of synthesis in a wide variety of sizes, shapes and porosities by methods similar to sol-gel processes. They are available in very small $(<30 \mathrm{~nm})$ sizes making them capable of crossing cell membranes, possess surfaces which are easily chemically modified and do not display swelling processes with changes in $\mathrm{pH}$ (Sahoo and Labhasetwar 2003). For example silica nanoparticles can be constructed with an anticancer drug entrapped within their cores (Roy et al 2003) which are stable in aqueous systems. Tumour cells take up these nanoparticles, which upon irradiation generate singlet oxygen which significantly damages the tumour cells.

\section{Commercial Systems}

There are a wide variety of commercial drug carrier agents available; we are only going to mention a few of these as a complete review would form an article itself.

\subsection{Polyanhydrides}

As previously mentioned, polyanhydrides are widely used as slow release agents due to the fact they biodegrade reproducibly and with no toxic by-products. Commercial products include materials such as Decapeptyl $\mathrm{SR}^{\circledR}$, manufactured by Ipsen Ltd which contains the active ingredient triptorelin acetate encapsulated in poly(lactide-coglycolide). This is utilised in the treatment of prostate cancer. Similar products 
include Lupron Depot ${ }^{\circledR}$ (TAP Pharmaceuticals Inc, active ingredient leuprolide acetate) and Sandostatin $\operatorname{LAR}^{\circledR}$ (Novartis, active ingredient octreotide acetate).

Nitroglycerin is a problematic drug due to its loss of tablet activity, often by volatilization of the active component (Markovich et al 1997). This can be avoided by incorporation of the nitroglycerin into an acrylic-based hydrogel which is then incorporated into a transdermal patch, as exemplified by products such as Deponit ${ }^{\circledR}$ (Schwarz Pharma), Minitran ${ }^{\circledR}$ (3M Pharma) and Nitrodisc ${ }^{\circledR}$ (G.D. Searle Company).

\section{Conclusions}

Drug delivery spans a wide variety of fields such as medicine, chemistry, biology and materials science. As fields such as genomics, proteomics and immunology progress, we will see these approaches being utilised to target drugs to specific sites with greater specificity. Further investigation into the transport of drugs across obstacles such as the blood-brain or air-blood barriers will enable development of simplified delivery systems for these compounds.

There are a wide number of potential techniques that would prove highly beneficial to medical practitioners and are currently being widely researched, some of which are listed below.

1. Biodegradable nanoparticles and methods for producing them.

2. More complex controlled release characteristics, for example in response to changing physiological conditions.

3. Delivery of large quantities of drugs in controlled and highly localised manner, for example to destroy a tumour without harming surrounding healthy tissue. 
4. Biodegradable coatings for implants, for example, for the release of agents that minimise rejection and/or promote healthy cell growth.

5. Functionalising the delivery system to enable selective targeting of sites via immunochemical response.

There are many future opportunities in this field, with one of the most exciting being the application of nanotechnology to drug delivery. The potential advantages associated with nanoparticles such as easy tailoring of properties such as size, often easy transition across cell membranes and potential site specificity ensures these approaches will be intensively studied. Problems to be overcome include the fact that these materials can be cytotoxic and often display poor stability in biochemical environments (Kaparissides et al 2006). However, these are challenges to be overcome and it can be seen that with the advances taking place in chemistry, materials, biology and other sciences that sooner or later they are likely to be achieved.

\section{Biosensors}

\subsection{History and format of biosensors}

The purpose of this chapter is to introduce the concept of using biological molecules as the selective recognition elements within biosensors. Most sensors consist of three principle components, as described below and shown in figure 4:

i. The first of these includes a receptor species, usually biological in origin such as an enzyme, antibody or DNA strand capable of recognising the analyte of interest with a high degree of selectivity; this is usually concurrent with a binding event between receptor and analyte. 
ii. The second component that must be present is a transducer, enabling the translation of the binding event into a measurable physical change; possible events include the generation of electrons, protons, an electrochemically active chemical species such as hydrogen peroxide or simple physical changes such as a change in conductivity, optical absorbance or fluorescence.

iii. Thirdly there must be inclusion of a method of measuring the change detected at the transducer and converting this into useful information.

There are several advantages associated with using biological molecules as the active recognition entity within a sensor. Usually they display unsurpassed selectivities; for example glucose oxidase will interact with glucose and no other sugar, and in this way will act as a highly selective receptor. In the case of glucose oxidase, the electrochemically inactive substrate glucose is oxidised to form gluconolactone along with the concurrent generation of the electroactive species hydrogen peroxide. Enzymes also generally display rapid turnover rates and this is often essential to (a) avoid saturation and (b) to allow sufficient generation of the active species in order to be detectable.

Antibodies bind solely to their antigens and achieve specificity via a complex series of multiple non-covalent bonds. Since the principle of immunoassay was first published by Yalow and Berson in 1959, there has been an exponential growth in both the range of analytes to which the technique has been successfully applied and the number of novel assay designs. Development of enzyme-labelled immunoanalytical techniques e.g. Enzyme-Linked Immuno Sorbent Assay (ELISA) has provided 
analytical tests without the safety risks associated with radiolabelling-based techniques.

The rapid measurement of analytes of clinical significance e.g. towards various disease markers would permit earlier intervention, which in a medical setting is frequently of utmost importance. There has been much research on the development of direct immunosensors that do not rely on the use of a detectable label. Such a system will lead to simpler assay formats and ideally lower times of detection. A reusable and rapid detection system would, moreover, allow for continuous real-time measurement, so helping to maintain optimal homeostatic conditions.

Unfortunately there are also some disadvantages related to the construction and use of biosensors. Often the biological species can either be extremely expensive or difficult to isolate in sufficient purity. Immobilisation of these species can lead to loss of activity and the presence of various chemical species in the test solution can also cause loss of activity e.g. enzymes can be easily poisoned by heavy metals. In biological samples such as blood or saliva, there can also be solutes that are electrochemically active and interfere with determinations of the target species. Again in physiological fluids such as blood, various species may be present which bind to the surface so causing fouling and loss of sensor response.

Antibody-antigen binding is based on multiple non-covalent interactions, and therefore there are no newly formed molecules, protons or electrons that are easily detectable, which has limited the development of direct antibody affinity-type sensors. Also affinity binding constants typically range from $10^{5}$ to $10^{11}$ mol. $1^{-1}$ meaning that 
the antibody-antigen binding event is often irreversible. As a consequence of this, many contemporary immunosensors are only of use for "single-shot" analyses and must be disposable in nature.

\subsection{Glucose biosensors}

A series of extensive reviews on biosensors and their history have been published elsewhere (Hall 1990, Eggins 1996, Wang 2001) and therefore only a brief history will be given here. Easily the most intensively researched area has been towards the development of glucose biosensors (Wang 2001, Newman et al 2004). The reason for this is the prevalence of diabetes which has become a world-wide public health problem. Diabetes represents an increasing epidemic with at the time of writing 170 million sufferers world-wide (World Health Organisation, www.who.org), and this is estimated to reach 300 million by 2045 (Newman et al 2004). Diabetes is related to a number of factors such as obesity and heart disease all of which make this disease one of the leading causes of death and disability in the world. The world market for biosensors is approximately $\$ 5 \mathrm{bn}$ with at the time of writing approximately $85 \%$ of the world commercial market for biosensors being for blood glucose monitoring (Newman et al 2004).

These factors have lead to the development of a number of inexpensive disposable electrochemical biosensors for glucose, incorporating glucose oxidase (GOD) bound immobilised at various electrodes. They are generally amperometric sensors, with electrodes polarised at a set potential; the oxidation or reduction of a chosen electroactive species at the surface will then lead to generation of a detectable current. The principle classes of glucose and other types of biosensors are described below. 


\subsection{First generation biosensors}

The first electrochemical glucose biosensor was based on an oxygen electrode (Clark and Lyons 1962). A film of immobilised glucose oxidase was laid down upon the oxygen electrode, which was overlaid with a semipermeable dialysis membrane. Upon exposure to glucose, the enzymatically catalysed oxidation reaction occurs, causing a localised consumption of oxygen.

\section{Glucose oxidase}

Eqn. 1. glucose $+\mathrm{O}_{2} \rightarrow$ Gluconolactone $+\mathrm{H}_{2} \mathrm{O}_{2}$.

This then leads to a drop in the current generated at the oxygen electrode. This device was subject to fluctuations caused by variable oxygen levels, however, further work (Updike and Hicks 1967) utilised two oxygen electrodes, one of which was coated with glucose oxidase. Measurement of differential current between the two electrodes served to cancel out these effects. The first commercial glucose analyzer was the Model 23 YSI analyzer, launched by the Yellow Spring Instrument Company in 1975 and based on the Clark electrode. This device was capable of measuring the glucose level in $25 \mathrm{ml}$ of whole blood.

The Clark oxygen electrode to monitor the depletion of oxygen caused by the oxidation of glucose is an example of a first generation biosensor, where the reaction causes depletion of an electrochemically active compound (oxygen) which can then be measured. An alternative technique is based on the amperometric monitoring of the product of the enzyme catalysed reaction, $\mathrm{H}_{2} \mathrm{O}_{2}$; Eqn. 2 . 
$+650 \mathrm{mV}$ vs $\mathrm{Ag} / \mathrm{AgCl}$

Eqn. 2. $\mathrm{H}_{2} \mathrm{O}_{2} \quad \rightarrow \quad 2 \mathrm{H}^{+}+\mathrm{O}_{2}+2 \mathrm{e}^{-}$

The results from these types of sensors can be affected by fluctuations in the ambient oxygen concentration or by the presence of electroactive species such as ascorbate, that are capable of being oxidised at $+650 \mathrm{mV}$ - giving rise to an erroneous result. Concentration of interferents at the electrode surface can be minimised by application of a permselective coating to the sensor, thereby reducing interference from electroactive species. Polymeric materials have led the way with materials such as the fluorinated ionomer Nafion (Turner and Sherwood 1994) and cellulose acetate (Maines and Vadgama 1993) being two of the most commonly used. A beneficial side-effect is that these materials can also confer a degree of biocompatibility. An alternative approach has been to electropolymerise suitable monomers to form protective coatings. 1,2-diaminobenzene (Myler et al 1997), for example, when deposited at the bioelectrode surface serves to both stabilise the electrode due to its inherent high biocompatibility whilst also imparting selective exclusion of interferents such as ascorbate.

The robustness of these types of sensors and, after suitable cleaning procedures, capability for multiple analyses makes them suitable for use within hospitals. However, diabetes as a condition requires regular monitoring of blood glucose levels, so hospital analysis is impractical for a normal lifestyle. The obvious solution has been the development of inexpensive home detection methods where the 
physiological sample, usually blood, can be analysed by the patient. The problems of cleaning the sensor are moreover negated by using disposable sensor strips.

\subsection{Second generation biosensors}

As shown earlier, the reaction of glucose oxidase with glucose gives rise to the formation of gluconolactone and the reduced form of the enzyme, which is then reoxidised by oxygen. Direct transfer of electrons from the electrode would circumnavigate this reaction, however the active site of glucose oxidase is encased in a protein sheath which inhibits this transfer. To facilitate this transfer, a suitable chemical species can be utilised to "shuttle" electrons back and forth between active site and electrode. This moiety, known as a mediator must react readily with the enzyme to avoid competition by ambient oxygen and in both its reduced and oxidised forms, be stable and preferably require as low an over-potential to be oxidised, as is feasible. This method sidesteps problems associated with detecting species such as oxygen or peroxide and also lowers the potential required for measurement of the enzyme catalysed reaction, thereby reducing the inference by redox active species present within the sample to be studied. A typical reaction scheme (Fig. 5) where a ferrocene compound acts as mediator is shown (Cass et al 1984). The first home glucose testing kit, the Exactech ${ }^{\circledR}$ glucose biosensor is based on this chemistry. The actual pen sized device is produced by Medisense ${ }^{\circledR}$ and utilises a disposable strip upon which a single drop of blood is placed. A wide range of glucose sensors (Wang 2001) based on this method have since become commercially available for home glucose testing. Further work has concentrated on reducing blood volumes and

produced devices such as the Pelikan ${ }^{\circledR}$ device which only require microlitre blood volumes (Newman et al 2004). 


\subsection{Third generation sensors}

Second generation biosensors are still somewhat limited in that they require use of a suitable mediator. Attempts have been made to circumvent this by developing methods to electronically directly connect or "wire" the enzyme to the electrode, thereby allowing simple electron transfer from the enzyme to the electrode without the requirement for a mediator. Although these types of devices have not as yet been developed commercially, they provide a possible alternative to mediated electron transfer.

Typical approaches involve the use of a polymeric coating which both immobilises the glucose oxidase and allows electron transfer to the electrode. One example is the use of polyvinyl pyridine where the synthetic polymer has been modified with a large number of osmium based electron transfer relays (Degani and Heller 1987, Ohara et al 1994). The polymer is co-immobilised at an electrode with glucose oxidase. When the enzyme reacts with glucose and is converted to the reduced form, the polymer allows facile transfer of electrons to convert it back to the oxidised form. Similar materials based on polyvinyl imidazole has also been utilised (Mano et al 2005). Glucose oxidase and other enzymes have also been immobilised on various electrodes by a variety of chemical and physical methods (Davis and Higson 2005).

Conducting polymers are especially suitable for the immobilisation of enzymes at electrode surfaces and have been reviewed in greater details elsewhere (Gerard et al 2002, Barisci et al 1994). A variety of monomers such as pyrrole or aniline can be electropolymerised on an electrode surface and under correct conditions form stable 
conductive films. If during this electropolymerisation process, enzymes are present in the solution they can be entrapped within the film during the deposition process, alternatively they can be adsorbed onto - or be chemically grafted to the film following deposition (Gerard et al 2002, Barisci et al 1994). The close association between the conductive polymer and the enzyme facilitates rapid electron transfer between the enzyme and an electrode surface.

One of the first methods involved the simple entrapment of enzymes such as glucose oxidase within polyaniline films (Cooper and Hall 1992). Our group has taken this process further, utilising both non-conductive and conductive polymers to fabricate arrays of conductive microelectrodes with entrapped biological molecules such as glucose oxidase (Barton et al 2004).

\section{Continuous Monitoring}

One problem with the current commercial biosensors is the invasive procedure, requiring frequent withdrawal of blood for testing which can be both tedious and painful. Devices which could be implanted within the human body would negate this aspect of glucose testing. Implantable sensors suitable for in vivo glucose monitoring require the device to be extremely small and show long-term stability (with minimal drift thereby removing the need for frequent calibration), display no oxygen dependency and also show high biocompatibility. The problem of biocompatibility has been the most elusive of these targets and as yet in vivo glucose sensors have only limited lifetimes. Effects on sensor performance include fouling by protein deposition on the surface or formation of fibrous tissue around the sensor (D'Orazio 2003) leading to loss of sensor performance. Rejection by the immune system or thrombus 
formation if used intravascularly can also degrade device performance and risk harm to the user.

Subcutaneously applied sensors have been developed (Bindra et al 1991, Henry 1998) which could monitor glucose concentrations and also be changed by the user.

However the effects of biofouling mean their use was limited to periods of 1-2 weeks. Again, fouling of the sensor and its effect on sensor performance is a major consideration. Attempts have been made to improve this using polymeric coatings. For example, a needle based electrochemical probe coated with Nafion has been developed (Moussy et al 1993) which is just $0.5 \mathrm{~mm}$ in diameter and can be inserted subcutaneously through an 18-gauge needle. Again in vivo measurements could be carried out over periods of 2 weeks.

A different approach has been to implant a microdialysis fibre into subcutaneous tissue and an iso-osmotic electrolyte solution pumped through the fibber. Glucose diffuses into the fibre and the electrolyte. If flow rates are kept constant the glucose concentration in the outflow from the fibre can be directly related to the glucose concentration in the interstitial fluid. Rapid changes in blood glucose concentration can be determined although there is a time delay, typically about 8 minutes (Janssen et al 1988) between changes in blood and interstitial fluid concentrations. The use of these probes combined with glucose biosensors to monitor the outflow have achieved 4-7 days continuous use for glucose monitoring in humans (Myerhoff et al 1992, Hashiguchi et al 1994). 
A subcutaneously implantable device, the $\mathrm{CGMS}^{\circledR}$ (Continuous Glucose Monitoring System) has been commercialized by Minimed (www.minimed.com). The glucose biosensor probe is inserted just beneath the skin, usually in the abdomen, and can be used to monitor glucose for up to $72 \mathrm{~h}$, with a reading every $5 \mathrm{~min}$. Traditional blood sampling of glucose is used to calibrate the device. Other data such as meal times and exercise periods can also be recorded using the device and all data then downloaded to a PC.

Non-invasive glucose monitoring systems are an active area of research. Techniques such as reverse iontophoresis, where a low electric current "pulls" interstitial fluid through the skin and onto a glucose biosensor negate the need for blood withdrawal. This technique has been utilised in the GlucoWatch ${ }^{\circledR}$ G2 ${ }^{\text {TM }}$ Biographer (Cygnus), a commercially available biosensor device for continuous glucose monitoring. The electric current pulls the interstitial fluid through the skin and collects it into two gel discs. These are in contact with an electrochemical glucose biosensor, which determines the glucose level. Again the device is calibrated using traditional blood testing. The device records glucose levels every 10 minutes for up to $13 \mathrm{~h}$ and can also sound an alert should the level stray outside predetermined levels. After 13 hours, the active part of the sensor (i.e. gel pads and biosensor) can be replaced and the device reused. $95 \%$ of GlucoWatch ${ }^{\circledR}$ G2TM Biographer readings are clinically accurate or acceptable (Newman et al 2004). However there are some problems, according to the company brochure the Glucowatch may be effected by excessive perspiration and can cause minor skin irritation. The Glucowatch is now supplied by Animas Technologies. 


\section{Future Needs}

This is a brief section just to highlight several potential future applications which, although not in the current marketplace, are the subjects of intensive research efforts.

\subsection{Point of care tests}

Although the biosensor market is dominated by glucose testing applications, there is great interest in developing simple tests for a wide variety of medical problems which can be easily applied by the patient and detect physical problems which are beginning to occur whilst still in an early stage, thereby enabling the patient to seek early medical help. An example of this is the Multisense ${ }^{\circledR}$ system developed by Oxford Biosensors (www.oxfordbiosensors.com) and soon to be commercialised which detects early markers of coronary heart disease. Heart disease is a major killer in the western world and the risk of developing this condition is known to be related to factors such as diet, smoking, weight and high blood cholesterol.

The Multisense ${ }^{\circledR}$ systems is based on a hand-held device with disposable electrochemical test strips. Each strip contains several sensors which individually sense for cholesterol (high density and low density lipoprotein) and triglycerides. Testing utilises a single drop of blood in the same manner as a glucose sensor. This will enable fast and simple screening, diagnosis and monitoring of patients who are at risk of heart disease.

\subsection{Artificial pancreas}

For many diabetes type 1 sufferers, life is a constant round of glucose testing and insulin injections. Externally worn insulin pumps have been developed, such as those 
by Medtronic (www.minimed.com). These have the advantage that they remove the need for injections by introducing insulin subcutaneously though a canella. When coupled together with a glucose biosensor, there is then the potential for the device to inject insulin "on demand". This was the first device of its type to receive FDA approval (Newman et al 2004). At present, current commercial devices usually can only be used for a few days at a time without maintenance and injection and sensing sites need to be changed regularly. An implanted device which automatically responds to changes in glucose levels would in effect act as an artificial pancreas and improve quality of life for many millions of people.

\subsection{Warfarin monitors}

Warfarin is routinely prescribed as an anti-coagulation agent for patients with an increased tendency for thrombosis or as prophylaxis in those individuals who have already formed a blood clot (thrombus) which required treatment. However warfarin when given in too high a dose inhibits natural clotting, leaving the patient subject to uncontrolled bleeding, both externally and gastrointestinal bleeding. At present, patients placed on warfarin require their blood to be regularly tested to ensure the clotting ability of their blood falls within required ranges (Poller et al 2003). This requires withdrawal of blood and testing within a laboratory environment. Since warfarin is one of the world's most proscribed drugs, a simple home test which could monitor warfarin levels or alternatively monitor blood clotting ability would have a huge global market.

\section{Conclusions}


As can be seen biosensors are a rapidly expanding field of research. Currently the market is dominated by glucose sensing. Many other applications such as cholesterol monitoring, monitors for various drug treatments and many other analytes are being intensively researched. It is likely that some of these applications will soon be commercialised. 


\section{Figures.}

Figure 1. Structures of (a) poly(glycolide), (b) poly(lactide).

Figure 2. Structures of (a) a micelle, (b) a vesicle.

Figure 3. Schematic structure of a fourth generation dendrimer.

Figure 4. Schematic of a biosensor.

Figure 5. The oxidation of glucose at an electrode, mediated by a ferrocene derivative. 
References:

Barisci J N, Conn C, Wallace G G (1996) 'Conducting Polymer Sensors', Trends Polym. Sci., 4, 301-311.

Barton A C, Collyer S D, Davis F, Gornall D D, Law K A, Lawrence E C D, Mills D W, Myler S, Pritchard J A, Thompson M, Higson S P J (2004) ' Sonochemically Fabricated Microelectrode Arrays for Biosensors Offering Widespread Applicability Part I', Biosens. Bioelec., 20, 328-337.

Bhadra D, Bhadra S, Jain S, Jain N K, (2003) ‘A PEGylated Dendritic Nanoparticulate Carrier of Fluorouracil', Int. J. Pharm. 257 111-124.

Bindra D, Zhang Y, Wilson G, (1991) 'Design and in vitro studies of a needle type glucose sensor for subcutaneous monitoring'. Anal. Chem. 63 1692-1696.

Brem H, Piantadosi S, Burger P C, Walker M, Selker R, Vick N A, Black K, Sisti M, Brem S, Mohr G, Muller P, Morawetz R, Schold S C (1995) 'Placebo-Controlled Trial of Safety and Efficacy of Intraoperative Controlled Delivery by Biodegradable Polymers of Chemotherapy for Recurrent Gliomas', Lancet 345 1008-1012.

Cass A E G, Davis G, Francis G D, Hill H A O, Aston W J, Higgins I J, Plotkin E V, Scott L D L, Turner A P F (1984) 'Ferrocene-Mediated Enzyme Electrode for Amperometric Determination of Glucose' Anal. Chem. 56, 667-671. 
Chen L, Remondetto G L, Subirade M, (2006) 'Food-based Protein Materials as Nutraceutical Delivery Systems’ Food. Sci. Tech. 17 272-283.

Cho C S, Han S Y, Ha J H, Kim S H, Lim D Y (1999) 'Clonazepam Release From Bioerodible Hydrogels Based on Semi-Interpenetrating Polymer Networks Composed of Poly(Epsilon-Caprolactone) and Poly(Ethylene Glycol) Macromer' Int J Pharm $181235-242$.

Clark A H, Kavanagh G M, Ross-Murphy S B (2001) 'Globular Protein GelationTheory and Experiment' Food Hydrocolloids, 15 383-400.

Clark L, Lyons C (1992) ' Electrode systems for continuous monitoring in cardiovascular surgery', Ann. NY Acad. Sci. 102, 29-45.

Cooper J C, Hall E A H (1992) ' Electrochemical response of an enzyme-loaded polyaniline film', Biosens. Bioelec., 7, 473-485.

Davis F, Higson S P J (2005) 'Structured Thin Films as Components in Biosensors', Biosens. Bioelec. 21, 1-20.

Degani Y, Heller A (1987) ' Direct Electrical Communication Between Chemically Modified Enzymes and Metal-Electrodes .1. Electron-Transfer from Glucose-Oxidase to Metal-Electrodes Via Electron Relays, Bound Covalently to the Enzyme', J. Phys. Chem., 91 1285-1289. 
Desai M P, Labhasetwar V, Walter E, Levy R J, Amidon G L (1997) ‘The Mechanism of Uptake of Biodegradable Microparticles in Caco-2 Cells is Size Dependent' Pharm. Res. 14 1568-1573.

Eggins B R, Biosensors, Chichester, Wiley, 1996.

El-Sayed M, Rhodes C A, Ginski M, Ghandehari H (2003) ‘Transport Mechanism(s) of Poly(amidoamine) Dendrimers Across Caco-2 Cell Monolayers, Int. J. Pharm. 265 $151-157$.

Gander B, Meinel L, Walter E, Merkle H P (2001) 'Polymers as a Platform for Drug Delivery: Reviewing our Current Portfolio on Poly (Lactide-Co-Glycolide) (PLGA) Microspheres' Chimia 55 212-217.

Gerard M, Chaubey A, Malhotra B D (2002) 'Application of conducting polymers to biosensors', Biosens. Bioelec. 17, 345-359.

Gombotz W R, Pettit D (1995) 'Biodegradable Polymers for Protein and Peptide Drug-Delivery’ Bioconjugate Chem 6 332-351.

Gupta U, Agashe H B, Asthana A, Jain N K (2006) 'Dendrimers: Novel Polymeric Nanoarchitectures for Solubility Enhancement' Biomacromolecules 7 649-658.

Guzman L A, Labhasetwar V, Song C X, Jang Y S, Lincoff A M, Levy R, Topol E J (1996) 'Local Intraluminal Infusion of Biodegradable Polymeric Nanoparticles - A 
Novel Approach for Prolonged Drug Delivery After Balloon Angioplasty’

Circulation, 94 1441-1448.

Hall E A C, Biosensors, Maidenhead, Open University Press, 1990.

Hashiguchi Y, Sakakida M, Nishida K, Uemura T, Kajiwara K, Shichiri M (1994)

'Development of a miniaturized glucose monitoring system by combining a needletype glucose sensor with microdialysis sampling method' Diabetes Care 17 387396.

Henry C (1998) 'Getting under the skin', Anal. Chem. 70 594A-598A.

Jansson PA, Fowelin J, Smith U, Lonnroth R (1988) 'Characterization by microdialysis of intercellular glucose level in subcutaneous tissue in humans' Am. J. Physiol. 255 E218-E220.

Jeong B, Bae Y H, Lee D S, Kim S W (1997) 'Biodegradable Block Copolymers as Injectable Drug-delivery Systems’ Nature 388 860-862.

Kaparissides C, Alexandridou S, Kotti K, Chaitidou S (2006) 'Recent Advances in Novel Drug Delivery Systems’ J. Nanotech. Online, 10.2240/azojono0111.

Kirpotin D, Park JW, Hong K, Zalipsky S, Li WL, Carter P, Benz CC, Papahadjopoulos D (1997) 'Sterically Modified anti-HER2 Immunoliposomews: 
Design and Targeting to Human Breast Cancer Cells in vitro', Biochemistry 36 6675.

Kojima C, Kono K. Maruyama K, Takagishi T (2000) 'Synthesis of Polyamidoamine Dendrimers Having Poly(ethylene glycol) Grafts and their Ability to Encapsulate Anticancer Drugs', Bioconjug. Chem. 11 910-917.

Kolhe, P, Misra E, Kannan, R M, Kannan S. Lieh-Lai M (2003) 'Drug Complexation, in vitro Release and Cellular Entry of Dendrimers and Hyperbranched Polymers', Int. J. Pharm. 259 143-160.

Langer R (1995) ‘Biomaterials and Biomedical Engineering’, Chem. Eng. Sci., 50(24) 4109-4121.

Langer R (1998) ‘Drug Delivery and Targeting’ Nature, 392[supp] 5-10.

Lasic D D, Vallner J J, Working P K (1999) ‘Sterically Stabilized Liposomes in Cancer Therapy and Gene Delivery', Curr. Opin. Mol. Ther., 1 177-185.

Liu X, Sun Q, Wang H, Zhang L, Wang J (2003) 'Microspheres of Corn Protein Zein for an Ivermectin Drug Delivery System', Biomaterials, 26 109-115.

Maeda H (2001) 'The Enhanced Permeability and Retention (EPR) Effect in Tumor Vasculature: The Key Role of Tumor-Selective Macromolecular Drug Targeting', Adv. Enz. Reg. 41 189-207. 
Maines A, Ashworth D, Vadgama P (1997) 'Diffusion Restricting Outer Membranes for Greatly Extended Linearity Measurements with Glucose Oxidase Enzyme Electrodes', Anal. Chim. Acta., 323, 223, 231.

Malik N, Evagorou E G, Duncan R (1999) 'Dendrimer-Platinate: a Novel Approach to Cancer Chemotherapy', Anticancer Drugs $10767-776$.

Maltais A, Remondetto G E, Gonsalves R, Subirade M (2005) 'Formation of Soy Protein Isolate Cold-Set Gels: Protein and Salt Effects', J. Food. Sci. 70 67-73.

Mano N, Mao F, Heller A (2005) 'On the Parameters Affecting the Characteristics of the "Wired" Glucose Oxidase Anode J. Electroanal. Chem., 574 347-357.

Markovich R J, Taylor A K, Rosen J (1997) 'Drug Migration from the Adhesive Matrix to the Polymer Film Laminate Facestock in a Transdermal Nitroglycerin System' J Pharm Biomed Anal 16(4) 651-660.

Moghimi S M, Hunter A C, Murray J C (2001) 'Long-Circulating and Target-Specific Nanoparticles: Theory to Practice' Pharmol. Rev. 53 283-318.

Moghimi S M, Szebeni J (2003) 'Stealth Liposomes and Long Circulating Nanoparticles: Critical Issues in Pharmacokinetics, Opsonization and Protein-Binding Properties', Prog. Lipid Res. 42 463-478. 
Moussy F, Harrison D J, O'Brien D W, Rajotte R V (1993) 'Performance of subcutaneously implanted needle-type glucose sensors employing a novel trilayer coating' Anal. Chem. 65 2072-2077.

Myerhoff C, Bischof F, Sternberg F, Zier H, Pfeiffer E F (1992) 'On line continuous monitoring of subcutaneous tissue glucose in men by combining portable glucose sensor with microdialysis' Diabetologia 35 1087-1092.

Myler S, Eaton S, Higson S P J (1997) ' Poly(o-phenylenediamine) ultra-thin polymer-film composite membranes for enzyme electrodes', Anal. Chim. Acta., 357, $55-61$.

Newman, J D, Tigwell L J, Turner A P F, Warner P J, 'Biosensors: A Clearer View, Biosensors 2004 - The 8th World Congress on Biosensors', Elsevier, New York, 2004.

Ohara T, Rajagopalan R, Heller A (1994) 'Wired Enzyme Electrodes for Amperometric Determination of Glucose or Lactate in the Presence of Interfering Substances', Anal. Chem. 66, 2451-2457.

Ottenbrite, R M, Frontiers in Biomedical Polymer Applications, Vol. 2, Lancaster, Technomic, 1999.

Park J W, Hong K, Kirpotin D, Papahadjopoulos D, Benz C C (1997) 'Immunoliposomes for Cancer Treatment' Adv. Pharmacol 40 399-435. 
Park JW, Kirpotin D, Hong K, Colbern G, Shalaby R, Shao Y, Meyer O, Nielsen U, Marks J, Benz CC, Papahadjopoulos D (1998) 'Anti-HER2 Immunoliposomes for Targeted Drug Delivery' Med. Chem. Res. 8 383-391.

Poller L, Keown M, Chauhan N, van den Besselaar A M H P, Tripodi A, Shiach C, Jespersen J (2003) 'Reliability of International Normalised Ratios from Two Point of Care Test Systems: Comparison with Conventional Methods, Brit. Med. J., 327 3032A.

Roco M C, Bainbridge W S (eds), (2002) Converging Technologies for Improving Human Performance. National Science Foundation Report. Kluwer Academic.

Roy I, Ohulchanskyy T Y, Pudavar H E, Bergey E J, Oseroff A R, Morgan J, Dougherty T J, Prasad P N (2003) 'Ceramic-Based Nanoparticles Entrapping WaterInsoluble Photosensitizing Anticancer Drugs: A Novel Drug-Carrier System for Photodynamic Therapy', J. Am. Chem. Soc, 125 7860-7865.

Sahoo S K, Labhasetwar V (2003) 'Nanotech Approaches to Drug Delivery and Imaging' Drug Discovery Today, 8 1112-1120.

Sokoloski T D, Royer G P, 'Drug Entrapment Within Native Albumin Beads' in Davis S S, Illum L, McVie J G, Tomlinson E, Microspheres and Drug Therapy, Pharmaceutical, Immunological and Medcial Aspects, Amsterdam Elsevier 2005, 295-307. 
Svenson S, Tomalia D A (2005) 'Commentary - Dendrimers in Biomedical Applications - Reflections on the Field' Adv. Drug. Delivery. Rev. 57 2106-2129.

Teijon J M, Trigo R M, Garcia O, Blanco M D (1997) 'Cytarabine Trapping in Poly (2-Hydroxyethyl Methacrylate) Hydrogels: Drug Delivery Studies’ Biomaterials 18 383-388.

Tomlinson E, Burger J J, 'Incorporation of Water Soluble Drugs in Albumin Microspheres' in Widder J, Green R Methods in Enzymology, New York, Academic $1985,27-43$.

Turner R B F, Sherwood C S (1994) ACS. Symp. Ser., 556, 211-221.

Updike S, Hicks G (1962) ' Electrode systems for continuous monitoring in cardiovascular surgery', Nature 214, 986.

Wang J (2001) 'Glucose Biosensors: 40 Years of Advances and Challenges', Electroanalysis, 13 983-988.

Yalow R S, Berson S A (1959) ' Assay of Plasma Insulin in Human Subjects by Immunological Methods', Nature. 184 1648-1649. 
<smiles>COC(=O)COC(=O)CC(C)(C)C</smiles>

a.<smiles>CC(OC(C)(C)C)C(=O)C(C)(C)C</smiles>

b.

Fig 1

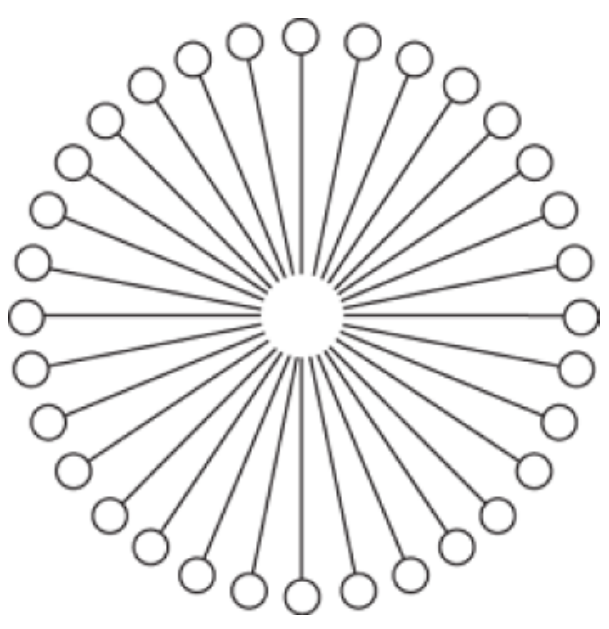

a.

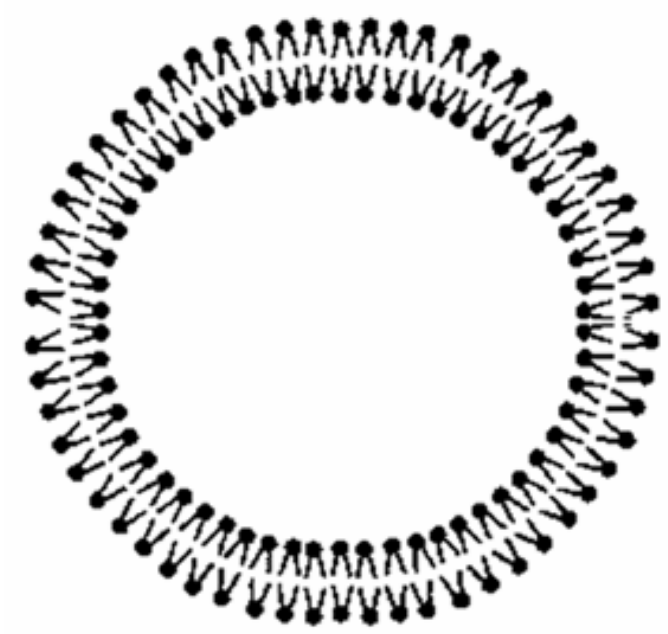

b.

Fig 2 


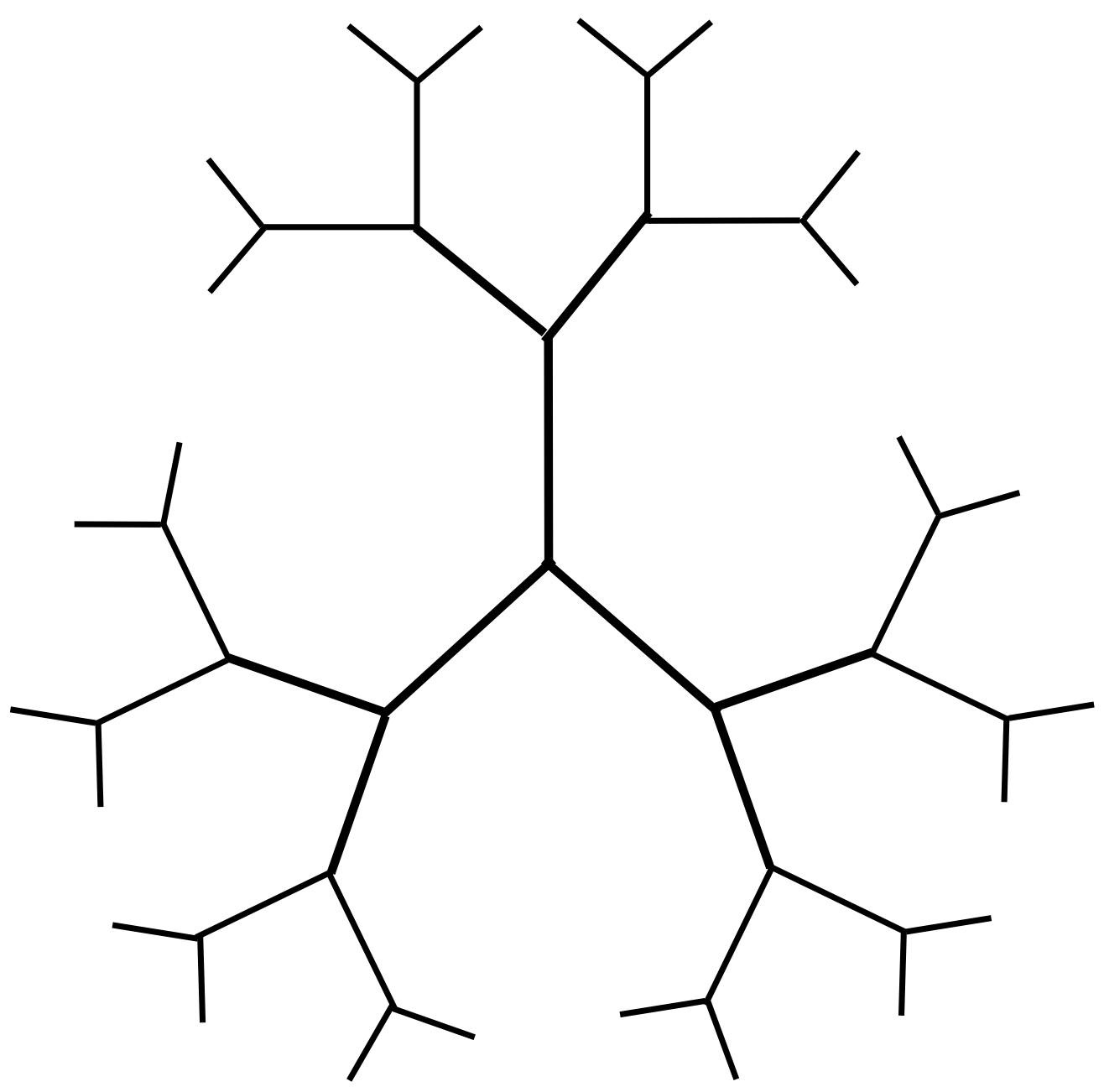

Fig 3

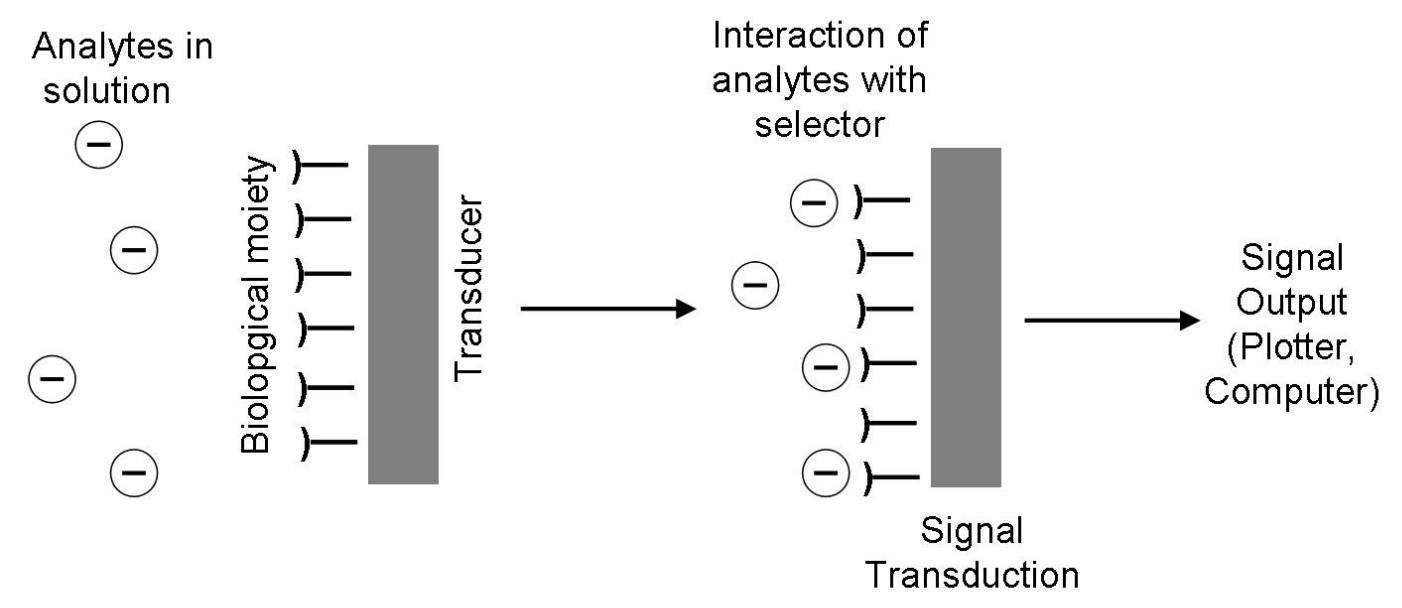

Fig 4 


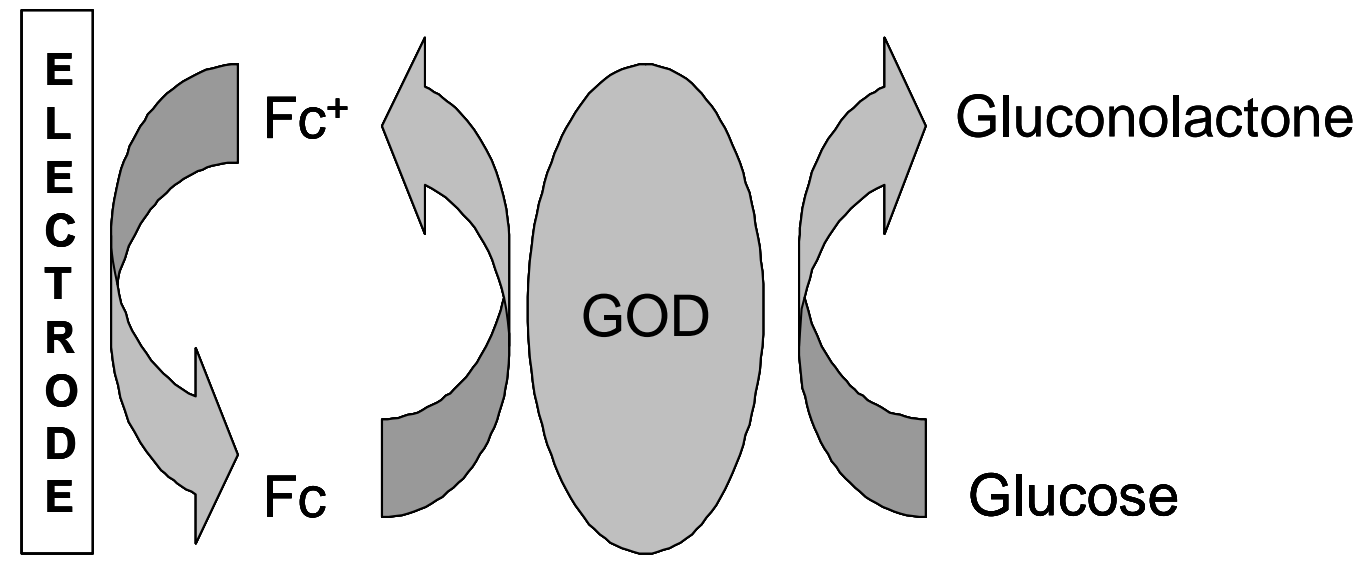

$\mathrm{Fc}=$ ferrocene derivative

Fig 5 